Originalveröffentlichung in: Patitz, Gabriele ; Grassegger, Gabriele ; Schinken, Karin (Hrsgg.): Natursteinsanierung Stuttgart 2019 : neue Natursteinrestaurierungsergebnisse und messtechnische Erfassungen sowie Sanierungsbeispiele ; Tagung am 15. März 2019 in Stuttgart, Stuttgart 2019, S. 7-25

Online-Veröffentlichung auf ART-Dok (2022), DOI: https://doi.org/10.11588/artdok.00007699

\title{
Das spätgotische Schlingrippengewölbe der Dresdner Schlosskapelle - Möglichkeiten, Methoden und Erkenntnisse der Wiederherstellung
}

von Stefan Bürger, Thomas Bauer und Jörg Lauterbach

Seit den 1980er Jahren plante man, die um 1550 gewölbte und 1737 zerstörte Schlosskapelle im Zuge des Wiederaufbaus des kriegszerstörten Dresdner Residenzschlosses zu rekonstruieren. Anhand eines Kupferstiches wurden drei methodisch sehr unterschiedliche Verfahren entwickelt, das Rippenwerk des ehemaligen Schlingrippengewölbes zu rekonstruieren. Bei der Neueinwölbung ist schlussendlich nicht versucht worden, den Kupferstich nachzubilden, sondern die spätmittelalterlich/frühneuzeitliche Vorgehensweise, wie damals ein solches Gewölbe entworfen und gebaut wurde. 


\section{Historisches zum Schloss, zur Schlosskapelle und zum Schlingrippengewölbe}

Mit vielen Um- und Ausbauphasen wuchs aus der frühen Burg und Wehranlage des 12. Jahrhunderts eine vielgestaltige, repräsentative Residenz heran, die bis in das frühe 20. Jahrhundert den herausragenden landesherrschaftlichen Mittelpunkt der Kurfürsten und später der Könige von Sachsen bildete. ' Während von den mittelalterlichen Bauphasen überirdisch nur Teile des östlichen Nordflügels und des Hausmannsturms (um 1400) erhalten blieben, haben sich in größerem Umfang Baukörper und -teile erhalten, die dem Ausbau zur Residenz im 16. Jahrhunderts zugehören.

1 Zur Baugeschichte von Schloss und Schlosskapelle mit weiterführender Literatur: Magirius, Heinrich: Die evangelische Schlosskapelle zu Dresden aus kunstgeschichtlicher Sicht, Altenburg 2009; Dülberg, Angelika; Oelsner, Norbert; Pohlack, Rosemarie: Das Dresdner Residenzschloss, Berlin 22013; Magirius, Heinrich: Schritte der Annäherung an ein fast verlorenes Baudenkmal, die evangelische Hofkapelle im Dresdner Residenzschloss. In: Das Schlingrippengewölbe der Schlosskapelle Dresden, hrsg. vom Staatsministerium der Finanzen des Freistaates Sachsen, Altenburg 2013, S. 8-13.
Unter Kurfürst Moritz von Sachsen wurde zwischen 1547 und 1556 der strategisch wichtige Ort am Elbübergang zu einer der bemerkenswertesten Renaissanceschlösser im Alten Reich ausgebaut. Ein kurz zuvor errichteter Westflügel wurde dafür niedergelegt und der Große Schlosshof um ein groBes Stück nach Westen erweitert. In diesem Zuge entstand der westlich an den Hausmannsturm anstoßende Nordflügel, der fortan die hohe Schlosskapelle beherbergen sollte. Diese Schlosskapelle war nicht nur privater Mess- und Andachtsraum für den Landesherrn, sondern als herausgehobener Repräsentationsort für Moritz konzipiert, um den neuen Stand als Kurfürst und im Zuge der Reformation die neue Rolle als summus episcopus des nunmehr lutherischen Fürstentums sichtbar zu machen. Das Schloss hatte dieser Vermittlungsfunktion weltlicher und geistlicher Machtansprüche zu genügen. Und die zwischen 1549 und 1556 geplante und gebaute Schlosskapelle bildete dabei den baukünstlerischen und medialen Mittelpunkt der Anlage. Es ist klar, dass daher jedes bau- oder bildkünstlerische Detail höchsten Ansprüchen genügen musste. Weniger bekannt ist, dass Schlingrippengewölbe, deren Verfahren und Formen aus der spätgotischen Bautradition hervorgingen, um und nach 1550 keinesfalls traditionell und veraltet

Bild 1

Schlosskapelle Dresden, Kupferstich David Conrad, aus: Christoph Bernhard, Geistreiches Gesangsbuch, Dresden 1676 [Deutsche Fotothek df_dat_0009796]

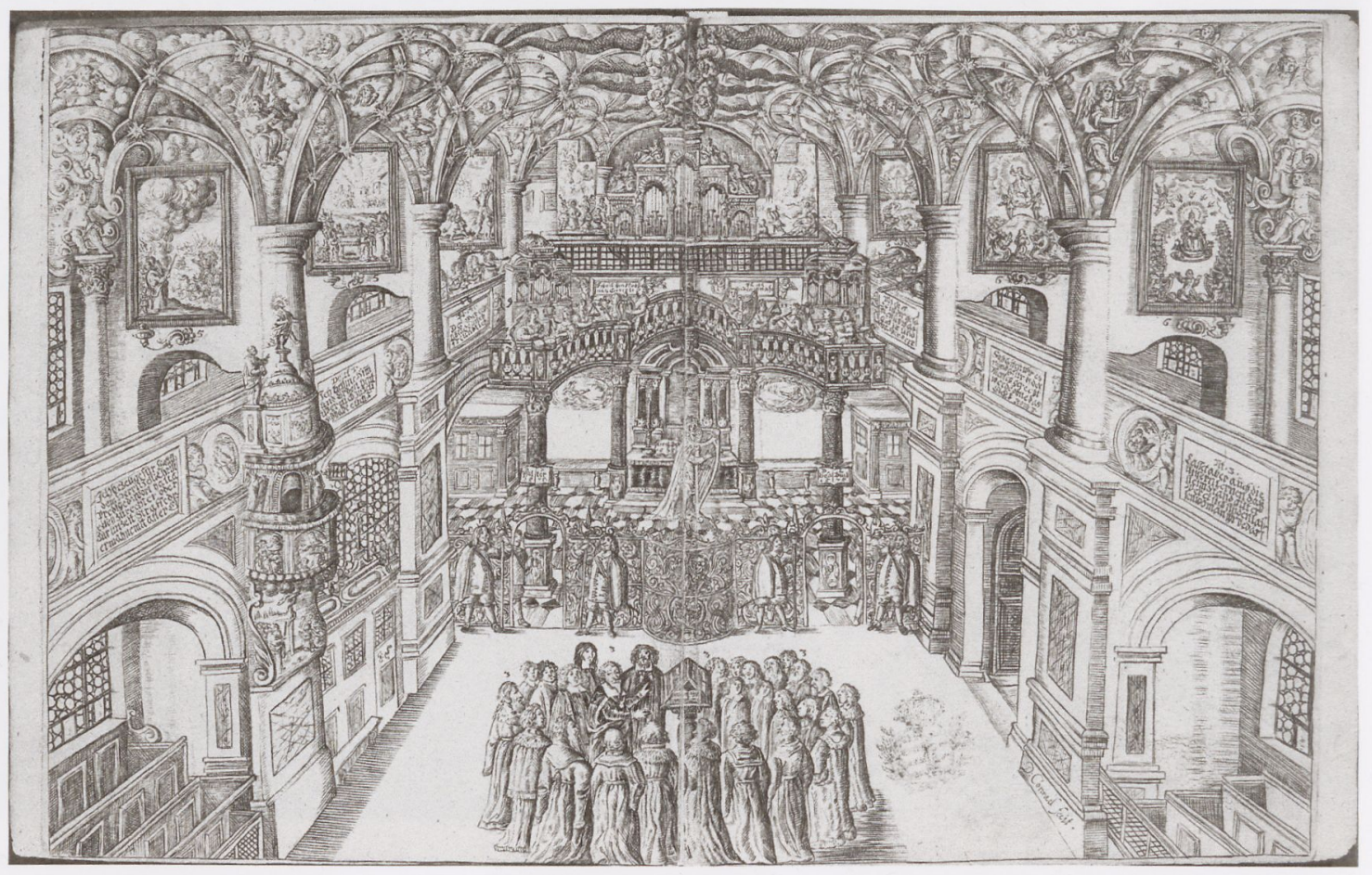


waren, sondern weiterhin innovativ und aktuell und damit auch dem Zeitstil und Zeitgeschmack zugehörig.

Über 150 Jahre bildete die Schlosskapelle das geistige und nicht zuletzt auch unter Hofkapellmeister Heinrich Schütz (1585-1672) das musikalische Zentrum des Landes (Bild 1). Die Bedeutung der protestantischen Schlosskapelle sank unter dem sächsischen Kurfürst Friedrich August II. (zugleich König August III. von Polen) herab. Um die polnische Königskrone erwerben zu können, war der Landesherr zum Katholizismus konvertiert. Die Kapelle wurde 1737 profaniert, zerstört, der Schlossflügel erheblich umgestaltet und fortan anderweitig genutzt.

\section{Bestand und Zustand nach 1945}

Die Kapelle als liturgischer und kunstvoll ausgestalteter Raum war bereits 1737 verloren. Der Sakralraum war profaniert, die Ausstattung und Einbauten entfernt, lediglich die Raumumfassung beibehalten und der gesamte Bereich für kleinteiligere Nutzungen umgebaut worden. Der westliche Nordflügel als gewachsener und umgestalteter Schlossteil und bauarchäologische Quelle erlebte abermals gravierende Verluste durch die schweren Zerstörungen des Zweiten Weltkrieges (Bild 2). Bereits 1947/1948 erfolgten erste Notsicherungen durch die Zwingerbauhütte. ${ }^{2}$

\section{Denkmalpflegezielsetzung 1978}

Die Ruine stand leidlich gesichert über Jahrzehnte offen, so dass nach 1945 Teile der zunächst noch erhaltenen Oberflächen verloren gingen. Durch die

2 Glaser, Gerhard: Zerstörung und Bemühungen um den Wiederaufbau. Eine Chronik und die denkmalpflegerische Zielstellung. In: Das Dresdner Schloß. Monument sächsischer Geschichte und Kultur. Eine Ausstellung der Aufbauleitung des Rates des Bezirkes Dresden, der Staatlichen Kunstsammlungen Dresden und des Instituts für Denkmalpflege Dresden, Ausstellungskatalog 1989, S. 99-119; Dülberg; Oelsner; Pohlack 2013 (wie Anm. 1), S. 22.
Umbauten des großen Kapellenraumes zu kleinteiligeren Innenraumnutzungen und nachfolgenden Veränderungen waren die Strukturen des ehemaligen Wandpfeilerraums geschwächt. Trotz aller Schwierigkeiten, die es zu DDR-Zeiten bereitete, ein solches Schloss als ehemaliges Zentrum feudaler Macht zu bewahren, gelang es lokalen Kräften, insbesondere dem Institut für Denkmalpflege, die Ruine vor Abriss oder Sprengung zu schützen und gar Pläne zum Wiederaufbau zu entwickeln (Bild 4). Bereits in den 1970er Jahren wurde dabei ins Auge gefasst, die ehemalige Schlosskapelle zu rekonstruieren. Ein ehemaliger höfischer Sakralraum sollte wiedererstehen, obwohl die politisch motivierten Sprengungen bedeutender Sakralbauten nur wenige Jahre zurücklagen.

\section{Bild 2}

Schloss Dresden Großer Hof mit Abbruch der (damals) nicht erhaltbaren Hofwand und beginnenden Neuaufbau [TT Fotoagentur und Verlag Siegfried und Thomas Thienel, 1986/87]
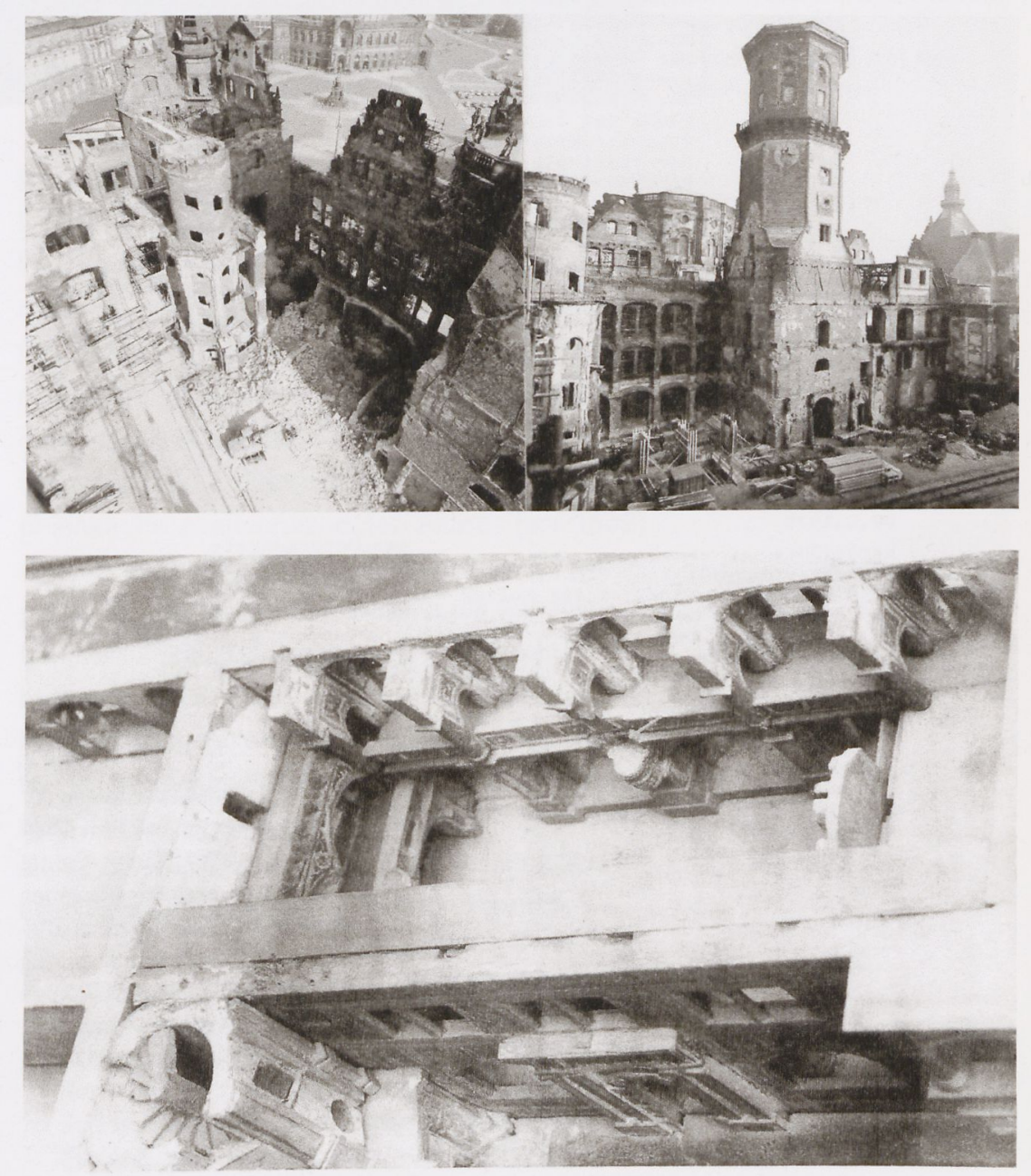

Bild 3

Modell des Schlosses um 1550 (Modell um 1960 zerstört), Einblick in die Schlosskapelle [Rat des Bezirkes Dresden/Institut für Denkmalpflege Dresden/Staatliche Kunstsammlungen Dresden (Hrsg.): Das Dresdner Schloss. Monument sächsischer Geschichte und Kultur, Dresden 1989; Bild 22 Seite 60] 


\section{Abschrift}

NUR FOR DEN DIENSTGEBRAUCH!
Institut für Denkmal.pflege

-Arbeitsstelle Dresden-

801 Dresden $A$. AugustusstraBe
Denkmalpflegezielstellung för das Dresdner SchloB

(Kurzfassung, Mindestforderungen)

1. AuBenbau : Wiederherstellung der straßenseitigen Fassaden einschließlich der Giebel in den Neorenaissanceformen von 1889-1902.

Wiederherstellung der Fassaden Großer und Kleiner SchloBhof itit Wendelsteinen und Altan in den Renaissanceformen von 1556 bzw, 1591.

Wiederherstellung des SchloBturmes in der Form von 1674 .

Auf die Beibehaltung und wiederherstellung der wirtschaft shoffassaden wird kein Wert golegt.

2. Innenräume: Beibehaltung bzw. Wiederherstellung sämtlicher Erdgeschoßgewölbe.

Erhaltung der Renalssanceraumstrukturen (enfilade) Im 1. und 2. OG des Westflügols.

Erhaltung der Raumform des Rtasensaalez im 2.OC Erhaltung der Raum

Erhaltung der barocken Raumstrukturen 1m 1. OG des

Wiederherstellung der zwe1geschossigen Raumstruktur der ehemaligen Schloßkapelle, nach Möglichkeft deren architektonische Rekonstruktion.

vollstanolge Restaurierung bzw. kekonstruktion der

Răune des Grünen Gewölbes.

Rekonstruktion der Engligchen Troppe einschlieblich der vestibülo.

Rekonstruktion der gotischen Räume im nörlichen latflügel.

Rekonstruktion des Porzellanzimmers 1m Turm, 2. OG

Rekonstruktion von Thronsal und Schlafzimmer August

des starken.

Rekonstruktion der Renaissance-Răume in 1. OG der

Hauptwache.

Nach Möglichkelt Rekonstruktion des groBen Ballsaales Im 3. OG, östlicher Nordflügel.

3. Archäologische

Grabungen: Freilegung des Kellerbereiches Ostfl.

Ausgrabung der Fundamente des Westflügels van 1471. Untersuchung der Gewölbe der 1. Augustusbrücke unter dem Georgenbau.

27.8 .1978

Verteller:

Bezirks-u.stadtleitung SED

Stadtarchitakt, Stadtrat für Kultur Leiter der Arbestsstelle

gez.Unterschrift

Prof.Dr.-Ing.Nadler Prof.Dr. - Ing. Nadie

TU. Prof. Dr. Milde Aufbauleitg.Staatsoper. Koll.Jeschke

Tu. Prof. Dr. Milde

If. B u Ve3 Denkmajpieqe Pro;

\section{Schlosswiederaufbaukonzept 1980er Jahre}

Von der vielbeachteten Aufbauleistung der Dresdner Semperoper konnte das Schloss unmittelbar profitieren. Im Zuge dieser positiven Wirkung der international beachteten Leistung wurde endgültig beschlossen, die Ruine zu bewahren und das Schloss als kulturhistorischen Mittelpunkt der Stadt wiederzubeleben. ${ }^{3}$ Die erhaltenen baulichen Reste, wertvolle Trümmerteile und Befunde wurden gesichert und unverzüglich mit Rohbauarbeiten begonnen. Gerade noch rechtzeitig konnte die Nordfassade des Schlossflügels im Bestand gerettet werden; die durch Feuer und Wetter zermürbte hofseitige Südfassade ließ sich jedoch nur noch abtragen und neu aufbauen. In dieser frühen Phase wurde sich besonders auf die nördlichen und westlichen Teile konzentriert - und damit auf den Bereich der ehemaligen Schlosskapelle. Innerhalb der kurzen Planung war bereits die Zielsetzung bzw. der Beschluss umgesetzt worden, die bereits 1737 verlorene Schlosskapelle zu rekonstruieren: Die einstigen Wandpfeiler wurden der Kubatur nach in den betonierten Rohbauteilen nachgebildet - dabei einige Befunde integriert - und mit innen die instabilen Fassadenabschnitte gesichert.

\section{Befunde und Dokumente zum ehemaligen Schlingrippengewölbe}

Bild 4

Denkmalpflegezielstellung für das Dresdner Schloss vom Chefkonservator Prof. Nadler, Institut

für Denkmalpflege Dresden [Nachlass VEB(B) Gesellschaftsbau Dresden, Sicherung

Schlosskomplex Dresden Gutachten und Stellungnahmen, Akte 06/62 17000$]$
Die Entscheidung zur Schlosskapellenwiederherstellung beruhte auf zahlreichen materiellen und immateriellen Zeugnissen (Bild 5). Die wenigen in der Substanz erhaltenen Reste hätten kaum gereicht, um eine solche Wiederherstellung zu begründen. Wichtiger war, dass schon die Kunstgeschichtsschreibung, insbesondere Cornelius Gurlitt, die ehemalige Schlosskapelle nachhaltig in

3 Glaser, Gerhard: Die denkmalpflegerische Konzeption zum Wiederaufbau des Residenzschlosses in Dresden. In: Denkmalpflege in Sachsen 1894-1994, Teil 1, Weimar 1997, S. 157-181; Coulin, Ludwig: Wiederaufbau der Schlosskapelle zwischen 1985 und 2013. In: Das Schlingrippengewölbe der Schlosskapelle Dresden, hrsg. vom Staatsministerium der Finanzen des Freistaates Sachsen, Altenburg 2013, S. 14-15. 


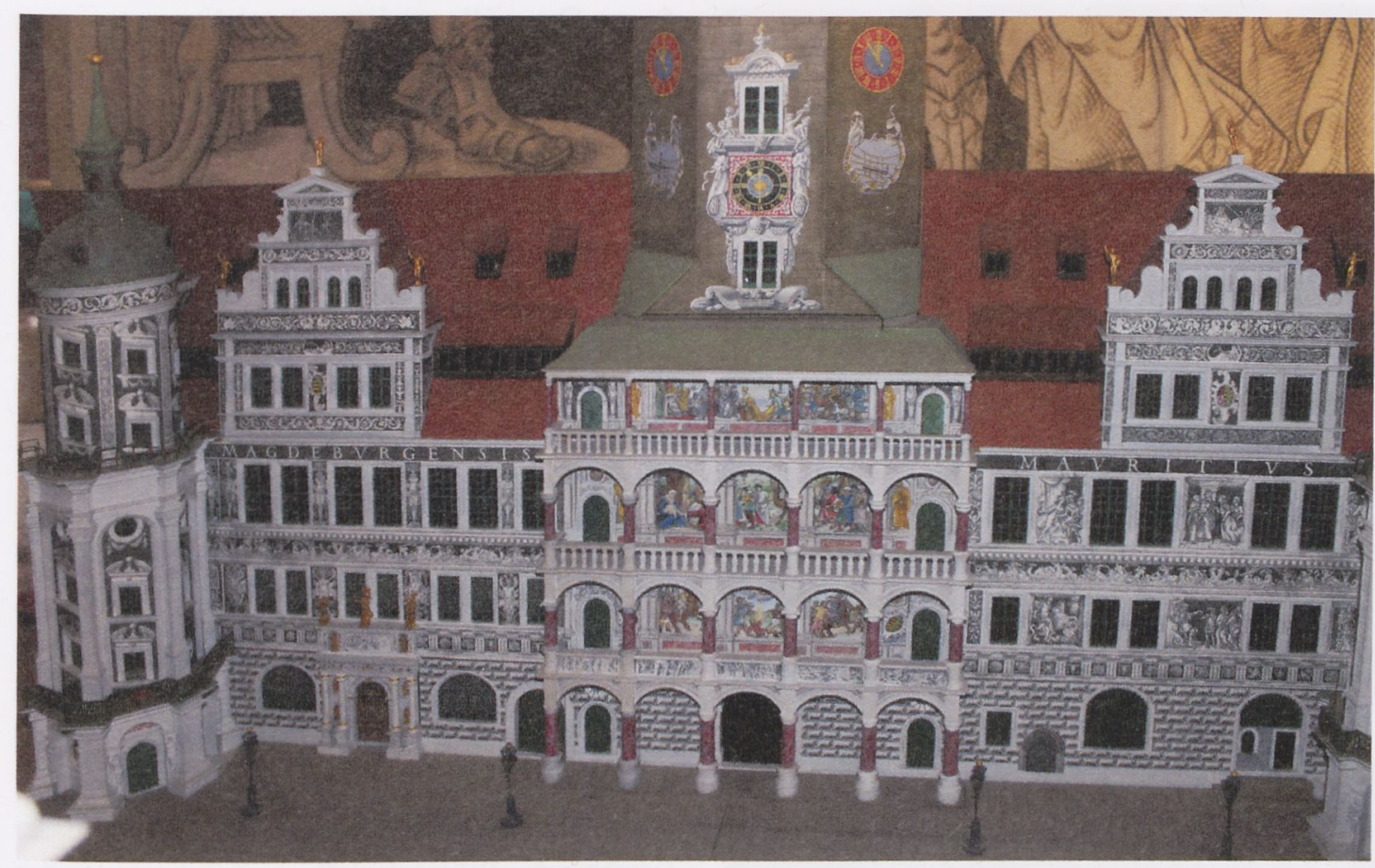

das kulturhistorische Gedächtnis der Stadt implementierte. ${ }^{4}$ Dabei fielen der Kapelle bzw. ihrer einstigen Bedeutung als hof- und kirchenmusikalisches Zentrum und dem erhaltenen und mehrfach translozierten Schlosskapellenportal als hochkarätiges Zeugnis nordalpiner Renaissancebaukunst nicht zu unterschätzende Rollen zu. ${ }^{5}$

Zu den greifbaren und für die Rekonstruktion bzw. spätere Wiederherstellung relevanten Zeugnissen und Befunden der Kapelle und des Gewölbes gehören neben bauarchäologischen Spuren an den erhalten Bauteilen, u. a. die Anschlüsse der nördlichen Wandpfeiler und der Gewölbeanfänger im

4 Gurlitt, Cornelius: Beschreibende Darstellung der älteren Bau- und Kunstdenkmäler des Königreichs Sachsen, Heft 21: Stadt Dresden, Dresden 1903, S. 144 -167, u. a. mit Bezug auf: Weck, Anton: Der Churfl. SächB. Weit beruffenen Residenz und Haupt Vestung Dresden beschreibt: und Vorstellung, Nürnberg 1680; ferner zur kunsthistorischen Bedeutung der Schlosskapelle u.a.: Krause, Hans-Joachim: Sächsische Schlosskapellen der Renaissance, Das Christliche Denkmal Heft 80, Berlin 1982.

5 Dazu: Schmidt, Eberhard: Der Gottesdienst am kurfürstlichen Hof zu Dresden. Ein Beitrag zur liturgischen Traditionsgeschichte von Johann Walter bis Heinrich Schütz, Berlin 1961; Dülberg, Angelica: „... weitaus die edelste Portalcomposition der ganzen deutschen Renaissance“. Zum Schlosskapellenportal des Dresdner Residenzschlosses. In: Jahrbuch des Landesamtes für Denkmalpflege Sachsen 2004, Beucha 2004, S. 52 - 80.
Bereich der ehemaligen Emporen, Abrissspuren vom Fußboden und Anfängerreste im Bereich der Westempore, Befunde zur bauzeitlichen Betstube im südwestlichen Kapellenbereich, einige 1986 aufgefundene und geborgene Rippenfragmente der ehemaligen Wölbung, sowie das Schlossmodell (Bild 3), das zu großen Teilen erhaltene Schlossportal und darüber hinaus kriegsversehrte Teile des in der Sophienkirche zweitverwendeten Altares (vor 1662) und des Taufsteins. ${ }^{6}$ Insbesondere sechs der geborgenen Rippenfragmente waren für die Rekonstruktionsvorhaben von Bedeutung, da sie einerseits bewiesen, dass es sich um ein Schlingrippengewölbe mit doppelt gekrümmten Bogenverläufen handelte, sich das genaue Rippenprofil abnehmen ließ und auch Aussagen zur einstigen Gestaltung bspw. der steinernen Schlangenleiber und zur einstmals kräftigen Farbfassung getroffen werden konnten.

6 Walther, Hans-Christoph: Dokumentation zur Schlosskapélle im Auftrag des SIB Dresden I, Dresden 2012; Rekonstruktionsgrundlage für die Wiederherstellung der Schlosskapelle. In: Das Schlingrippengewölbe der Schlosskapelle Dresden, hrsg. vom Staatsministerium der Finanzen des Freistaates Sachsen, Altenburg 2013, S. 19-26; Bürger, Stefan; Anwand, Jens-Uwe: Das Schlingrippengewölbe - Zur Methode der Formfindung. In: Das Schlingrippengewölbe der Schlosskapelle Dresden, hrsg. vom Staatsministerium der Finanzen des Freistaates Sachsen, Altenburg 2013, S. 39-40. Walther, Hans-Christoph: Quellen und Befunde als

\section{Bild 5}

Renaissanceschloss Dresden, Zustand im letzten Drittel des 16. Jahrhunderts, Modell aus dem Jahre 1989 von Franz Brettschneider, Siegfried Winderlich und Martin Wolf [Ausstellung 1989 „Das Dresdner Schloss. Monument sächsischer Geschichte und Kultur" der Aufbauleitung des Rates des Bezirkes Dresden, der Staatlichen Kunstsammlungen Dresden und des Instituts für Denkmalpflege Dresden in den Räumen des Grünen Gewölbes; Foto vom Modell s. Titel Ausstellungsband, hier Neuaufnahme: Autoren] 
Das mit gängigen, vor allem stilistischen Methoden der Zeit konzipierte Gewölbe sollte mit Natur- und Backsteinen realisiert werden. Das Modell zeigt eine Gewölbegeometrie, bei der die Rippen auf einen kreuzgratgewölbeartigen Wölbgrund appliziert worden wären. Von Vorteil hätte sein können, dass die Kreuzgewölbeform leicht konzipierbar und statisch beherrschbar geworden wäre. Die Rippen wären als sekundäre Elemente behandelt worden; man hätte immerhin eine bildhafte Annäherung an eine spätgotische Gewölbeform erreicht. Nachteilig bzw. schwerer beherrschbar wären die exakte Geometrie und die Verläufe der Rippen gewesen. Zahlreiche Versuche und Entscheidungen hätten notwendig sein müssen, um sich anhand der sekundären Bildquellen der einstigen Gestaltung des Raumes anzunähern. Annäherungen über Stilempfinden und Raumwirkungen wären die maßgeblich methodischen Grundlagen für das bildkünstlerische Verfahren dieser Rekonstruktion gewesen im Sinne einer Nachbildung eines räumlichen $\mathrm{Ge}$ bildes anhand eines zweidimensionalen Abbildes. ${ }^{8}$

\section{Gewölbewiederherstellungs- konzepte 2008}

Im Zuge der schrittweisen Auf- und Ausbaumaßnahmen der Nachwendezeit war beabsichtigt, die Paraderäume im zweiten Obergeschoss und auch des westlichen Nordflügels wiederherzustellen. Im Vorfeld wurde entschieden, über die Gestaltung des Schlosskapellengewölbes neu nachzudenken, da es später schwer bis unmöglich sein würde, unter dem Boden der Paraderäume massive Deckenbzw. Wölbarbeiten durchzuführen. Inzwischen war aus architekturhistorischer Sicht hinsichtlich des spätmittelalterlichen Gewölbebaus der Kenntnisstand weiter fortgeschritten und es war klar, dass eine solche Raumgestaltung und -wirkung primär von der Gestaltung des Rippenwerks auszugehen habe. Den Rippen als primären Gestaltungselementen war bei der Konzeption mehr Aufmerksam-

8 Dazu: Bürger, Stefan: Re-Konstruktion eines Prozesses. Der Nachbau des Schlingrippengewölbes in der Dresdner Schlosskapelle. In: Popp, Dietmar; Korduba, Piotr (Hrsg.): Re-Konstruktionen. Stadt, Raum, Museum, 2019 (Betrag in Vorbereitung zum Druck).

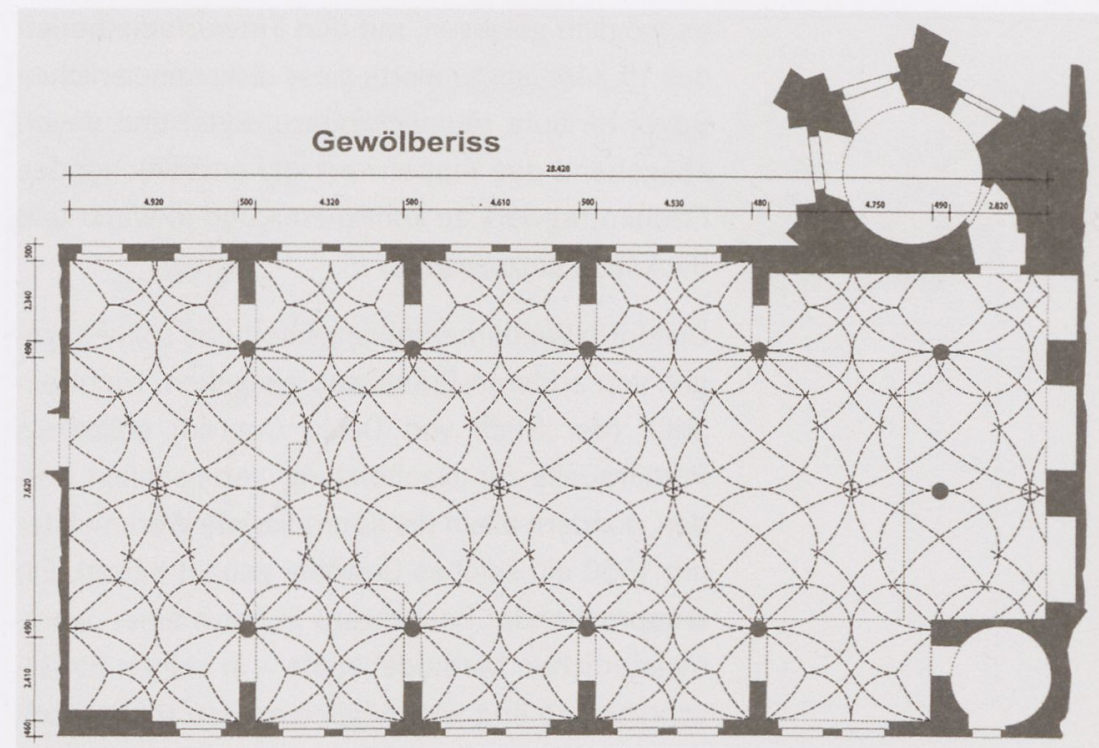

keit zu schenken, als der Struktur der Wölbschale bzw. des Kappenwerks.

Von der nachbildenden Methode wurde Abstand genommen und versucht, eine neue, zweite Methode zu entwickeln. In einem neuen, nunmehr zweiten Anlauf extrahierte das beauftragte Architekturbüro Anwand aus dem Kupferstich David Conrads durch Projektion und Spiegelung der erkennbaren Rippenverläufe in den Grundriss ein Lineament. Durch den ,Rückbau' bildkünstlerischer Bestandteile und perspektivischer Bildmittel konnte mit dieser ,geometrischen Methode' ein System von Bogenlinien ,re'-konstruiert werden, die zusammengenommen als Wölbriss dienen sollten, um davon ausgehend das Rippen- und Kappenwerk zu konzipieren (Bild 8). ${ }^{9}$ Von Vorteil war, dass die Wiederherstellungsmethode der Prämisse folgte, dass ein solches Schlingrippengewölbe von der geometrischen Konstruktion der Rippenlinien her entworfen und gebaut worden war. Allerdings war vor dem Hintergrund der Kenntnis, wie ein figuriertes Gewölbe räumlich über einem planimetrischen Wölbriss ausgetragen würde, abzusehen, dass ein solches Lineament mit wechselnden Radien und einer atypischen Geometrie und Kappenbeschaffenheit sich ebenfalls einer spätgotischen Gewölbegestaltung nur annähern würde. Keinesfalls wäre

9 Bürger, Stefan; Anwand, Jens-Uwe: Das Schlingrippengewölbe - Zur Methode der Formfindung. In: Das Schlingrippengewölbe der Schlosskapelle Dresden, hrsg. vom Staatsministerium der Finanzen des Freistaates Sachsen, Altenburg 2013, S. 42 - 47

\section{Bild 8}

Wölbriss zur 2. Methode - die verworfen wurde - mit jenen aus dem Kupferstich extrahierten und in den Grundriss projizierten Rippenverläufen; zu sehen sind eine Vielzahl verwirrender Bogenlinien mit unterschiedlichen Radien; die Bogenverläufe sind zwar so genau als möglich aus der Bildvorlage rekonstruiert, doch ungeeignet für eine Bogenaustragung, letztlich weil es dem Zeichner aus seiner perspektivischen Ansicht des Gewölbes nicht möglich oder wichtig war. die Konstruktionen geometrisch genau wiederzugeben.

[Architekturbüro Anwand, Jens Teupel, Archiv Stefan Bürger] 
es möglich gewesen, mit den Entwurfsmethoden des 15./16. Jahrhunderts diese diskontinuierlichen Bogenverläufe räumlich auszutragen und davon ausgehend das Rippenwerk als entsprechendes Flächentragwerk zu konzipieren und in Werk- und Backstein umzusetzen.

Die dritte Wiederherstellungsmethode sollte dagegen von anderen Prämissen ausgehen. Nicht ein ,Bild' (der Stich von David Conrad) sollte als Primärquelle zur ,Nachbildung' veranschlagt werden, sondern allein die Kenntnis, wie Werkmeister um 1550 ein solches Gewölbe gebaut hätten. Ein entsprechender Bauprozess in Form einer, werkmeisterlichen Methode' sollte - in seinen Einzelschritten so eng wie möglich an den theoretisch rekonstruierten Entwurfs- und Bauphasen werkmeisterlicher Handhabungen - ,nachgebildet' werden. Am Ende der Prozesse sollte bestenfalls ein Gewölbe(bild) entstehen, das den verfügbaren Bildquellen so weit wie möglich entsprach. Da diese prozess-rekonstruierende Methode, was die Formfindung anbelangt, auf einfachen Prämissen und leicht kontrollierbaren Verfahrensschritten beruhen würde, wurde 2008 innerhalb kurzer Zeit entschieden, das Schlingrippengewölbe in einer Rohbauform - d.h. ohne bildkünstlerische Anteile, auch ohne Putz und Farbfassung - wiederherzustellen. ${ }^{10}$

\section{Rekonstruktion des Rippen- werks, Entwurfsprozess}

Die spätgotische Wölbkunst basiert auf sehr einfachen Prinzipien bzw. Handhabungen. Für viele Verfahrensschritte des Entwerfens und Bauens war es günstig, wenn alle Rippen über einheitliche Radien verfügten. Im Prinzip folgen die Verfahrensweisen für den Entwurf und Bau figurierter Gewölbe den Grundlagen zum Kreuzrippengewölbebau: Von den Anfängern ausgehend verlaufen die Rippenprofile auf einer Bogenlinie zum höchsten Scheitelpunkt im Gewölbe, der bei Kreuzgewölben in der Regel den Jochmittelpunkt bildet. Bei Kreuzgewölben sind Anfänger und Scheitelpunkt (Schlussstein) durch den Rippenbogen direkt mit-

10 Die Projektleitung seitens des Auftraggebers oblag Holger Krause (SIB); leitender Architekt war Jens-Uwe Anwand (Architekturbüro Anwand). einander verbunden. Bei figurierten Gewölben sind Anfänger und Scheitelpunkt ebenfalls durch einen Bogen (den sog. Prinzipalbogen) miteinander verbunden. Doch verlaufen diese Bögen nicht in direkter Linie von den Anfängern aufsteigend zu den höchsten Scheitelpunkten, sondern folgend den gewinkelten (im Forschungsdiskurs auch alternativ gesehen als abzuwickelnden) Linienverläufen des Wölbrisses.

Sollte sich das Rippenwerk aus doppelt gekrümmten Bogenrippen (gewundene Reihungen, Schlingrippen) zusammenfügen, bedeutete das, dass diese sich im Grundriss als gleichförmig große Kreislinien abzeichnen mussten (Bild 9); und die über diesen Kreislinien aufgerissenen Prinzipalbögen folgten im räumlichen Verlauf ihren geschwungenen Formen (Abwicklung). Über jeder Bogenlinie im Grundriss schwingt sich somit eine Bogenlinie im Aufriss empor. Aber nicht jedes Liniensystem im Grundriss (bspw. das der zweiten Methode) lässt sich mit Prinzipalbögen im Aufriss sinnvoll austragen.

Das Verfahren basiert dabei auf festen Regeln, die enge Grenzen setzen. Dies hat den Vorteil, dass sich das Verfahren leicht handhaben und kontrollieren lässt. Aber die Grund- und Aufrissmaße und Befunde des Raumes müssen zu den diesbezüglichen Parametern und Prämissen passen. D.h. wenn sich ein Wölbriss aus gleichgroßen Kreis(segment)en zusammenfügen soll, dann muss die Grundrissgeometrie bei Figurationen, die in der Fläche aus gleichgroßen Kreisen bestehen ", sich zwangsläufig aus Quadrat(teil)en zusammensetzen. ${ }^{12}$ Allerdings entsprach ein Jochformat der

11 Anders ist dies bei sog. Schleifensternfigurationen, deren Scheitelfigur geometrisch auf einem Sechseck basiert - eingeschrieben in einem Umkreis im Maß der kleineren Jochbreite; vgl. Nachweise zu den Konstruktionsverfahren Benedikt Rieds für den Wladislawsaal, die Schneckenstiege und das Wladislaworatorium auf dem Prager Hradschin (Norbert Nußbaum; Thomas Bauer; Jörg Lauterbach, div. Beiträge in INSITU) sowie zu Jacob Haylmanns Gewölben für St. Annen in Annaberg und Mariä Himmelfahrt in Most/Brüx (Vortrag Thomas Bauer; Jörg Lauterbach auf der Tagung „Werkmeister im Konflikt - Der Annaberger Hüttenstreit und andere Streitfälle im Bauwesen des 15. und frühen 16. Jahrhunderts“, 28.09.2018 Dresden, Publikation in Vorbereitung)

12 Bürger; Anwand 2013 (wie Anm. 9), S. 52 - 63. 

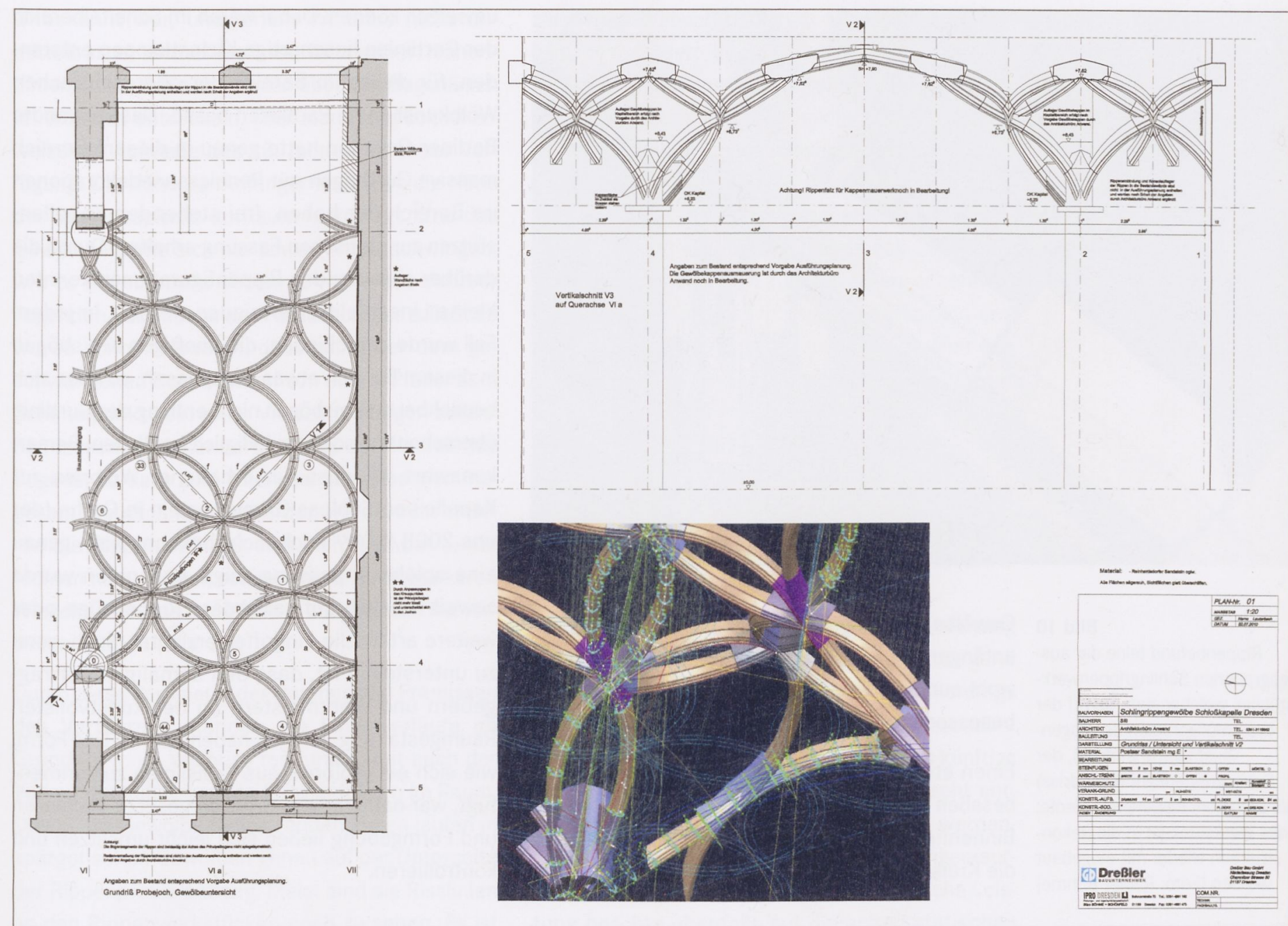

Schlosskapelle keinem solchen Regelmaß. Diesbezüglich war zu berücksichtigen, dass die damaligen Werkmeister in der Lage waren, baukünstlerischen Mehrwert zu schaffen, in dem sie die Wölbrissdispositionen und -gestaltungen von den Grundrissen ablösten: D.h. die Wölbrisse eines gewölbten Joches besaßen nicht selten Maße, die sich nicht mit den Grundrissen deckten. Im Falle der Schlosskapelle sollten sich die Wölbjoche entlang der Jochgrenzen überlappen und zwar in dem $\mathrm{Ma} ß$ der Mauerstärke der dortigen Wandpfeiler. Dadurch überschnitten sich die Figurationen und bewirkten im Bereich der Anfänger über den tuskischen Säulen eine artifizielle Formverdichtung durch sich über- und unterschneidende Rippenzüge. Die Wölbrissgeometrie beruhte in einem Mittelschiffjoch - also der Länge nach über ein solches Grundrissjoch hinausreichend - auf einem Quadratraster im Maßverhältnis von 2:3. Genau dies ließ sich durch Nachmessungen im Raum und in den Rissen des 18. Jahrhunderts nachprüfen und bestätigen. In dieses Raster ließ sich ein einfaches System aus Kreislinien einzeichnen.

Für solche Schlingrippengewölbe gab es eine lange Vorentwicklung: Entsprechende Wölbungen haben sich in der Kirche in Neusohl/Banská Bystrica, im niederösterreichischen Landhaus (Landtag) in Wien und im Zwickauer Gewandhaus erhalten oder sind, wie das mit Dresden eng verwandte, leider kriegszerstörte Gewölbe der ehemaligen Erasmuskapelle im Berliner Stadtschloss in Vorkriegsfotos überliefert. An solchen formverwandten Gewölben lieBen sich später zahlreiche Detailgestaltungen der Rippenverläufe und Kappenstrukturen untersuchen und ggf. überprüfen. ${ }^{13}$ Das ehemalige Berliner

13 Bauer, Thomas; Lauterbach, Jörg: Die Schlingrippen der Gewölbe - Erasmuskapelle Berlin, Rotbergkapelle Basler Münster, Landhauskapelle Wien, Eleemosynariuskapelle Banská Bystrica, Ratssaal Bunzlau/Bolesławiec, Rathaus Löwenberg/Lwówek Śląski, Dresden 2011

\section{Bild 9}

Steintechnische Werkplanung Jörg Lauterbach; Thomas Bauer (für Dreßler Bau GmbH/IPRO) auf der Basis der Entwicklung der unteren Rippenmittellinien und dem Abgriff der Befundrippen von $A B$ Anwand/Stefan Bürger [Autoren, Planarchiv bauer lauterbach] 
Unbekannt war, und einem Wölbriss nicht unmittelbar zu entnehmen ist, in welcher Höhendimension sich die Kreislinien über den Bogenlinien im Wölbriss entwickeln. Hier standen glücklicherweise die Aufmaße des 18. Jahrhunderts zur Verfügung: Die Höhe der Anfänger war durch die Höhe der Säulenkapitelle im Raum festgelegt. Und das Gewölbe konnte sich nur soweit emporschwingen, wie es die ebenfalls bekannte Höhe des darüber liegenden Fußbodens der Paradezimmer zuließ. Es wurde schnell deutlich, dass sich über dem geschwungenen Bogenverlauf von einem Pfeiler zum Scheitelpunkt kein voller Viertelkreisbogen aufziehen lassen wird, sondern jeweils nur ein Teil des Bogens, so dass zum einen der Gewölbestich insgesamt deutlich kleiner werden würde, zum anderen die Rippenanfänger sich nicht aus der Senkrechten herauslösen, sondern bereits mit stärkeren Winkelmaßen aus den Pfeilern und Wänden hervortreten würden (Bild 11).

Durch diese Parameter des Raumes und Prämissen des Verfahrens standen die Linienverläufe im Grund- bzw. im Wölbriss fest und damit auch der Verlauf der aufsteigenden Prinzipalbögen im Raum. Diese, das Gewölbe definierenden Linien laufen in spätgotischen Gewölben genau auf der Unterseite der Rippenprofile entlang. Meist sind die Risslinien an den Rippenwerkstücken noch zu sehen. Es ist somit nur noch notwendig, das Rippenprofil (deren Rippenprofil-Mittelsenkrechte/Rippenprofillotachse immer tangential zum Kreisbogen verlaufend) an den jeweils flankierenden Fugenflächen der Rippenstücke anzureißen und diese Profilflanken mit Reißziehlehre oder freihändig (so die Erfahrungen in Dresden) steinmetzseitig auszuarbeiten. Die kompliziert erscheinenden Rippendurchdringungen, Hinter- und Überschneidungen entstehen somit nicht durch aufwändige Detailentwürfe, sondern unweigerlich durch das Einhalten einer sehr einfachen Entwurfslogik entsprechend der rationalen Prinzipalbogengeometrie. Das Rippenprofil war durch die aufgefundenen Rippenstücke bekannt.

Anmerkung: Zu sehen war, dass die Rippenfunde doppelte Krümmungen aufweisen. Allerdings waren die Rippenfunde kaum geeignet, die Raum- und Wölbgeometrie zu rekonstruieren. Die Bogensteine reichten nur bedingt aus, um die Radien genau zu ermitteln. Zudem besteht durch das Zusammenspiel von gebogener Grundrisslinie und gebogener Aufrisslinie kaum eine Möglichkeit exakt vorzubestimmen, wo sich das Bogenstück einst befunden hat - bzw. umgekehrt. Bei unterschiedlichen Annahmen, wo sich das Rippenstück im Gewölbe befunden hat, ergeben sich zwangsläufig unterschiedliche Maßgaben für ein entsprechend zu rekonstruierendes Gewölbe. Es musste also erst ein Verfahren rekonstruiert und ein Gewölbe entworfen und gebaut werden, um zu prüfen, ob das Rippenstück zum Gewölbe passt - und wenn ja, an welcher Stelle (Bild 10).

\section{Herstellung des Probejochs}

Für die Fragen der Formbildung und -gestaltung wäre der Bau eines Probejoches nicht zwingend notwendig gewesen (Bilder 12 und 13). Allerdings stellte das avisierte Gewölbebauprojekt besondere Herausforderungen an die Statik, denn das künftige Tragwerk musste nach deutschem Baurecht entworfen, geprüft, genehmigt und errichtet werden. Die 2017 mit dem Ulrich-Finsterwalder-Ingenieurbaupreis ausgezeichnete ingenieurtechnische Leistung beruhte ebenfalls auf einfachen statischen Vorüberlegungen und Prämissen. ${ }^{14}$ Es wurde zunächst davon ausgegangen, dass ein hölzernes Lehrgerüst, das Rippenwerk aus Werksteinen und das Kappenwerk aus Backsteinen jeweils drei separate Tragwerke bilden. Das Lehrgerüst kann sich selbst tragen und auch die Last der zu versetzenden Rippensteine. Das Rippenwerk ist in der Lage, zunächst noch im Verbund mit dem hölzernen Lehrgerüst stehend, die Last des nachfolgend aufgemauerten Kappenwerks aufzunehmen und Lasten auf das aufgehende Mauerwerk der Wände und Pfeiler abzuleiten (Bild 14). Während das Holzgerüst später entfernt wird, verbleibt das Rippenwerk als ,steinernes Lehrgerüst' im Gewölbe. Inwieweit es für die eigenständige Tragfähigkeit des

14 Bürger, Stefan; Kröning, Matthias: Das Rippengewölbe als komplexes Tragwerk. In: Das Schlingrippengewölbe der Schlosskapelle Dresden, hrsg. vom Staatsministerium der Finanzen des Freistaates Sachsen, Altenburg 2013, S. $96-107$. 
Bild 12

Entwurf zum Probejoch: Entwicklung der Rippenfiguration mit sog. Drahtmodell der Rippenbasislinien (Mittellinie auf der Stegunterseite) auf

Grundlage der Befunde von

Raummodell, Kupferstich, Befunden an Schlingrippenfragmenten und Höhen der Wölbanfänger durch Architekturbüro Anwand und Stefan Bürger [Architekturbüro Anwand, Archiv Stefan Bürger]

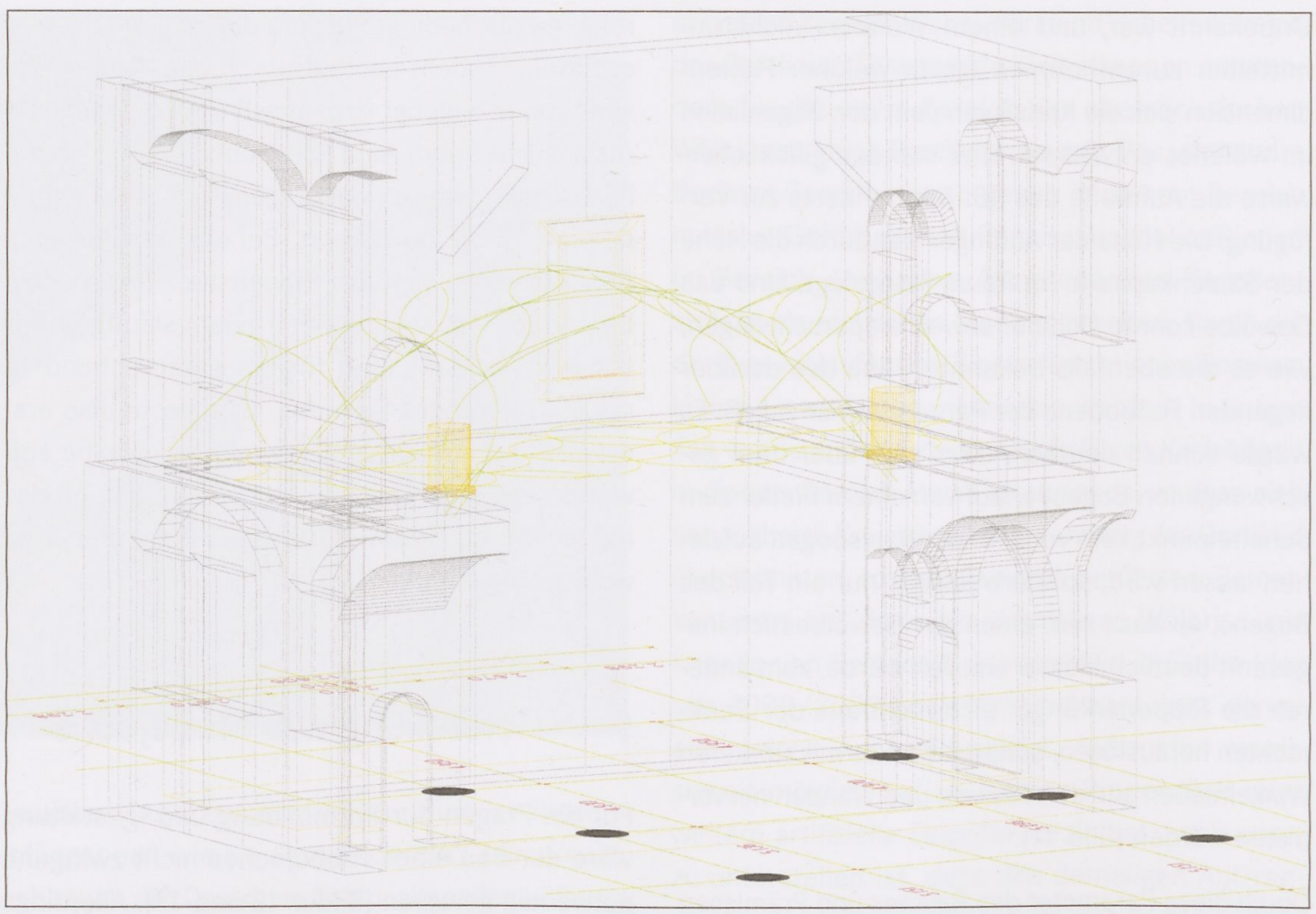

Bild 13

Entwurf zum Probejoch: Entwicklung eines 3D-modellierten Rippenkörpermodells

inkl. aller Krümmungen als steintechnische Werkplanung für die Ausführung des Probejoches durch Jörg Lauterbach und Thomas Bauer für Dreßler Bau GmbH/IPRO auf Grundlage des Figurationsentwurfs von Architekturbüro Anwand und Stefan Bürger [Autoren, Planarchiv bauer lauterbach]

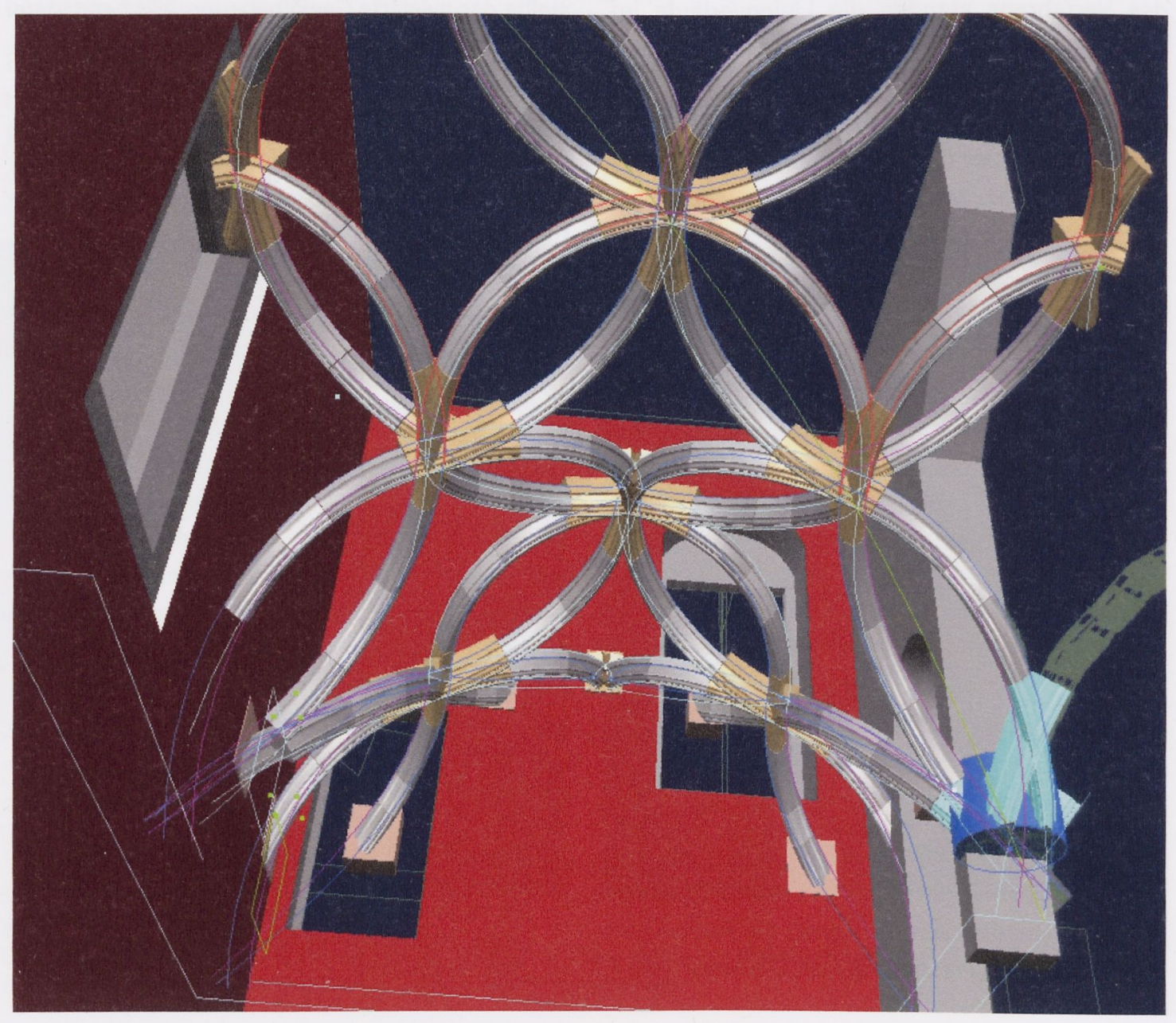


Kappenwerks im letztendlichen Lastzustand zwingend notwendig wäre, ist in der bisherigen Forschung umstritten und wird teils kontrovers diskutiert. ${ }^{15}$

Dem Ingenieurbüro Kröning-Ulbrich-Schröter oblag es, diese Vorstellungen und entsprechend formalen Prämissen in statische Modelle zur Wölbung zu überführen. Das Lehrgerüst wurde von Thomas Bauer und Christian Willich entwickelt, konstruiert und statisch nachgewiesen.

Im Ergebnis zeigte sich, nachdem insbesondere durch Matthias Kröning alle bekannten Lastabtragungsmodelle berücksichtigt und simuliert worden waren, dass alle computergestützten Modellierungen, statischen Berechnungen und Lastsimulationen nicht ausreichen würden, um den Lastabtrag eines schlingrippengewölbten Joches rechnerisch nachweisen zu können und somit eine Genehmigung für das Tragwerk zu erreichen. Aus diesem Grunde wurde entschieden, ein Probejoch (aus technologischen Gründen auf ehemaliger Emporenhöhe) im Maßstab 1:1 herzustellen, dabei alle Bauphasen einmal zu durchlaufen, um ein $\mathrm{Ge}-$ wölbe zu errichten, das sich anschließend einem Belastungstest unterziehen ließe, um die Tragfähigkeit zu prüfen und nachzuweisen.

Bei dem Belastungsversuch ${ }^{16}$ wurden im Probejoch - in welchem bei einem Drittel keine Rippen verbaut worden waren, sondern die Wölbung allein aus Kappenmauerwerk bestand - u. a. das Spannungs-Dehnungsverhalten untersucht und durch das Experiment belegt, dass die Wölbschale vom Kappenmauerwerk allein getragen wird, da messtechnisch in Bereichen mit und ohne Rippen identische Werte erfasst wurden (Bild 15).

15 Fehr, Götz: Benedikt Ried, München 1961, S. 27-29; Müller, Werner; Quien, Norbert: Virtuelle Steinmetzkunst der österreichischen und böhmisch-sächsischen Spätgotik, Petersberg 2005, S. 8-11; Bürger/Kröning 2013 (wie Anm. 14), bes. S. $105-106$

16 Kröning, Matthias; Opitz, Heinz: Rechnerische und experimentelle Untersuchungen zum Tragverhalten des Schlingrippengewölbes in der Schützkapelle des Dresdner Schlosses, In: Manfred Curbach, Heinz Opitz, Silke Scheerer, Torsten Hampel (Hrsg.): 7. Symposium Experimentelle Untersuchungen von Baukonstruktionen Schriftenreihe Konstruktiver Ingenieurbau der Fakultät Bauingenieurwesen der Technischen Universität Dresden, Heft 32, 2013, S. $155-169$

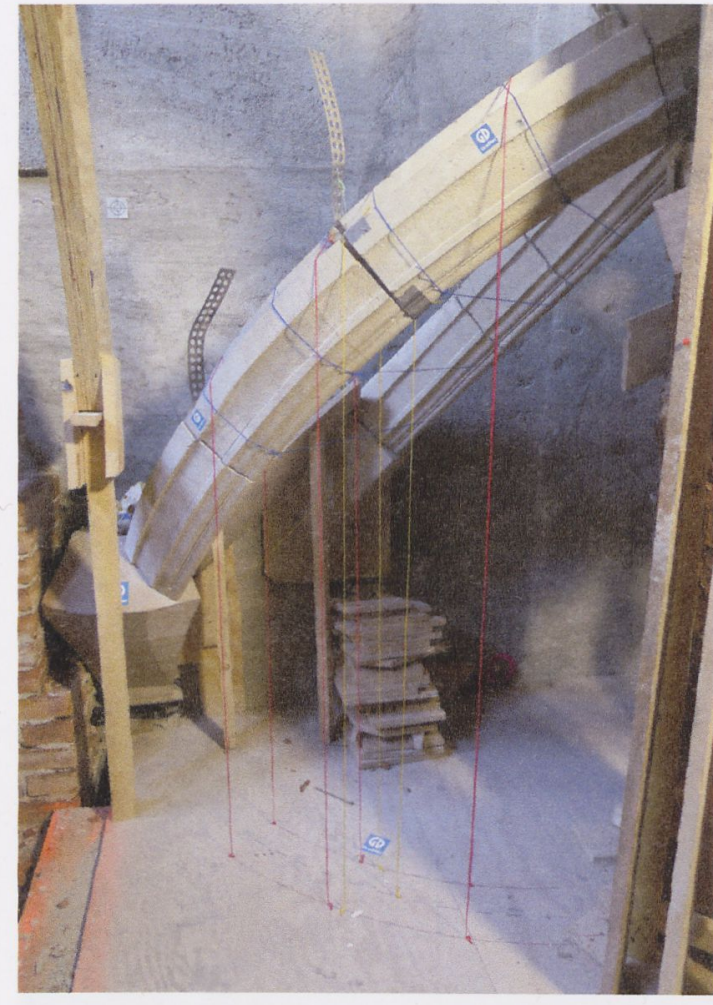

Bild 14

Schlingrippenherleitung am Probejoch, sog. Strickmodell von Thomas Bauer; Jörg Lauterbach: Aufriss der Schlingrippenfigur im Grundriss (rote Stricke), geometrische Lagebezug der Rippenfuge (gelbe Stricke) und Höhenlagen der Bogenaustragung (blaue Stricke) [Strickmodell Thomas Bauer; Jörg Lauterbach; Foto: M. Ventas]
Mit dem Entwurf und Aufzug der Prinzipalbögen wurde am Probejoch aber nicht nur die Formgebung des Rippenwerks und seiner Bogenwerkstücke festgelegt, sondern zugleich auch der geometrischen Bogenaustragung folgend die Höhenpunkte für das hölzerne Lehrgerüst. Anders als man dies von (bspw. römischen und romanischen) Gewölben mit Vollschalungen oder von (gotischen) Lehrgerüsten zur Unterstützung der Rippenbögen kennt, ist es für figurierte Gewölbe mit kleinteiligen Rippenlängen und Kappenflächen nicht notwendig und auch nicht sinnvoll, aufwändige Schalungen und Lehrbogenkonstruktionen herzustellen. ${ }^{17}$

17 Bauer, Thomas; Lauterbach, Jörg: Die Ausführung des Schlingrippengewölbes der Schlosskapelle Dresden, Abschnitt 1.1. „Lehrgerüstkonzept“, In: Das Schlingrippengewölbe der Schlosskapelle Dresden, hrsg. vom Staatsministerium der Finanzen des Freistaates Sachsen, Altenburg 2013, S. 128 -132; sowie: Bauer, Thomas; Lauterbach, Jörg: Rekonstruktion des Schlingrippengewölbes der Schlosskapelle Dresden - Teil II: Lehrgerüste, Bauzustände und Verformung der Wölbung, In: Bundesverband öffentlich bestellter und vereidigter sowie qualifizierter Sachverständiger e.V. (Hrsg.) Der Sachverständige. Fachzeitschrift für Sachverständige, Kammern, Gerichte und Behörden, 43. Jg. Heft 5/2016, S. $119-128$. 

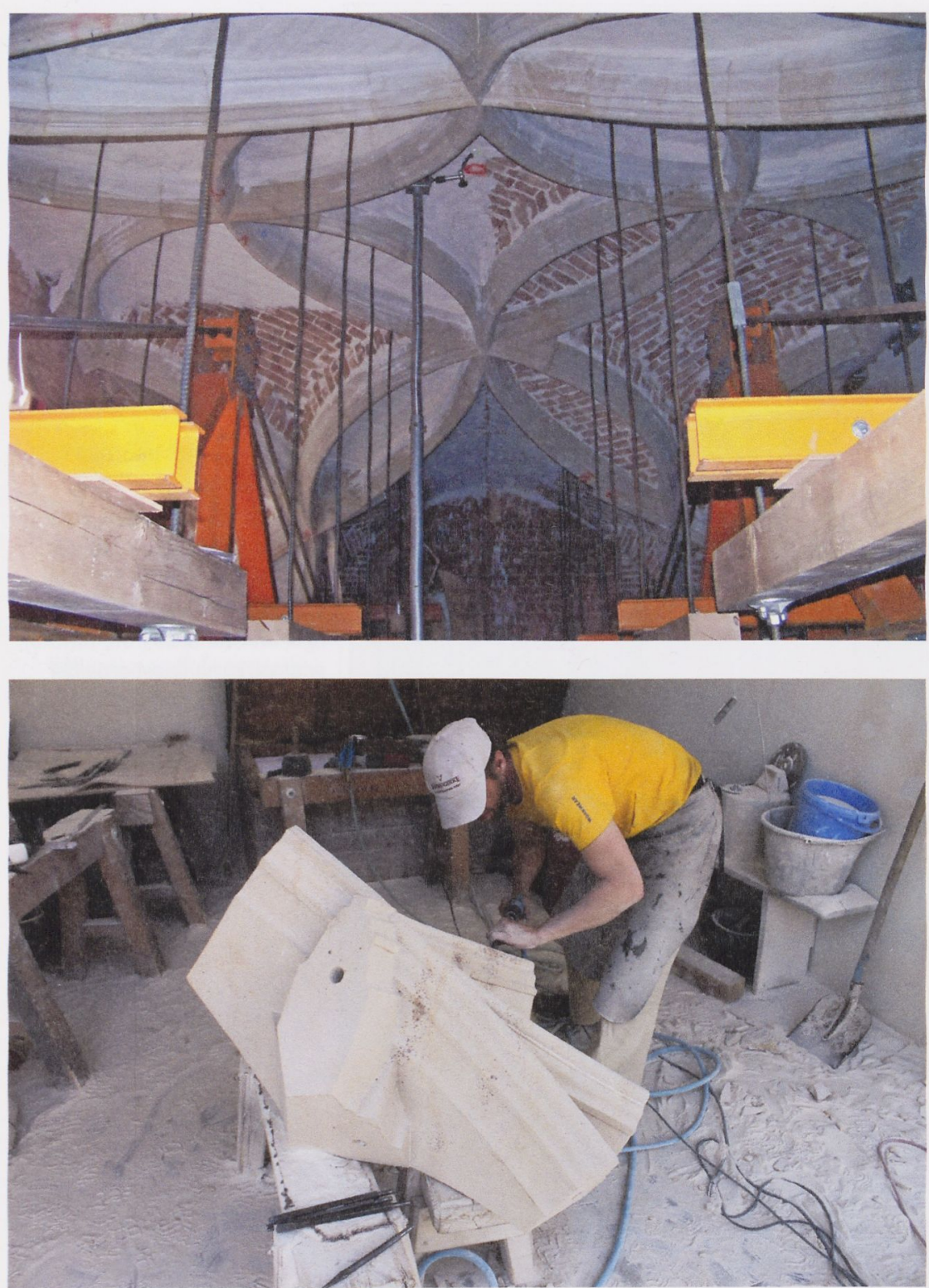

Bild 15 Die Erkenntnisse zur Konzipierung eines LehrProbejoch mit Belastungsversuch des Otto-Mohr-Labors der Technischen Universität Dresden über Untersuchungen zum Spannungs-Dehnungsverlauf der Wölbung [Foto: Autoren]

Bild 16

Steinmetzarbeit am Schlingrippenknoten auf dem Werkplatz der Firma Fuchs+Girke Bau- und Denkmalpflege $\mathrm{GmbH}$

in Ottendorf-Okrilla mittels sechsseitiger Schablonen der steintechnischen Werkplanung

[Foto: Thomas Bauer] werden die Teilstrecken - der Bogenabschnitte von Rippenknoten(-kreuzung) zu Rippenknoten - von einem Anfänger bis zum Scheitelpunkt auf einer geraden Grundlinie nacheinander abgetragen. Danach wird über dieser aus Teilabschnitten kumulierten Grundlinie (im 16. Jh. Zwerchlinie genannt) ein Kreisbogen (zumeist Viertelkreis) geschlagen. Bei einem Viertelkreisbogen dient der Endpunkt der Grundlinie (gemäß dem Scheitelpunkt des Gewölbes) als Mittelpunkt der Kreislinie des Prinzipalbogens. Über den Anfangs- bzw. Endpunkten der kumulierten Streckenabschnitte werden lotrechte Linien aufgezogen, die sich mit dem Prinzipalbogen schneiden. Die jeweiligen Längen der Senkrechten zwischen der Grundlinie und dem Kreisbogen der Bogenaustragung ergeben dann die Höhen der jeweiligen Lage des betreffenden Kreuzungssteines bzw. davon ableitend die Höhe des Lehrgerüstes an diesem Punkt. Dieses Verfahren lässt sich mit einfachen Zeichenwerkzeugen über maßstäblich verkleinerten Wölb- und Lehrgerüstentwürfen simulieren, wie auch im Maßstab 1:1 auf dem Reißboden beim Herstellen und Aufrichten des Lehrgerüstes umsetzen.

Ein wichtiger Moment, um die Richtigkeit aller Annahmen zu prüfen, war das Einpassen eines der großen Fundstücke, um zu sehen, ob dieses zum hergestellten Gewölbe passt (Bild 10). Tatsächlich ließ sich der Werkstein in das Rippensystem einfügen, genau an der Position im Bereich nahe der Anfänger, die man vorab (aufgrund der abgefasten Rückseite) gemutmaßt hatte. Dieses Ergebnis war ein weiteres und wichtiges Indiz und Beweisstück dahingehend, dass sich die Herstellung des Gewölbes einer Wiederherstellung des ehemaligen Gewölbes annäherte.

Beim Versatz wurden dann - anders als die landläufige Vorstellung - alle Kreuzungs- und Schlusssteine (d. h. die in den Quellen genannten Schlosssteine, die mehr als zwei Rippenstücke koppeln) auf diesen Stammhölzern zuerst versetzt. Dabei müssen alle Kreuzpunktsteine zunächst ohne Kontakt zueinander gemäß jenen durch Bogenaustragung ermittelten Höhenmaßen exakt versetzt, d. h. ihrer Höhe nach aufgerichtet werden (Bild 17). Beim Arbeiten am Probejoch ergab sich zunächst die Schwierigkeit, da die Kreuzpunktsteine an ihren Unterseiten kunstvolle Rippenprofilierungen, dage- 
gen keine waagerechten Basislinien oder -flächen und zudem materialseitige Schwächungen aufweisen, dass sich die Werkstücke nicht direkt auf die Holzstämme des Lehrgerüstes setzen ließen. Annahmen neuzeitlicher Quellen stellten sich als nicht praktikabel heraus. ${ }^{19}$ Diesbezüglich halfen Untersuchungen an vergleichbaren Gewölben bspw. der St. Barbarakirche in Kuttenberg/Kutná Hora und St. Marien in Pirna weiter, wo wölboberseitig Rippenkreuzungssteine erkennbar sind, die zwischen den Rippenschenkeln Steinzwickel mit waagerechter Ober- und wohl auch Unterseite besitzen. Von dieser Beobachtung ausgehend, war es möglich, die Stammhölzer des Lehrgerüstes im Kopfbereich durch kleinere ,Zwickelstützen' in der Weise aufzustocken, dass sich auf diesen Stützen das jeweilige Werkstück absetzen ließ. Da kein Lehrgerüst aus dem 15./16. Jahrhundert mehr existiert, lässt sich diese Methode kaum nachprüfen: Doch wie angedeutet, wurde unterstellt, dass die einfachste Methode der historisch gebräuchlichen am nächsten kommen müsste. Eine andere Untersuchung nahm die an spätgotischen Rippenwerken bzw. deren Kreuzpunktsteinen nicht selten zu findenden Metalldübel in den Blick, an denen später oftmals Wappen, Sterne o. a. appliziert wurden. Es ist denkbar, das diese Bolzen wenn, dann zumeist an allen Kreuzpunkten eines Gewölbes zu finden - im Versatzprozess zunächst zur drehbaren Arretierung auf den Stammhölzern dienten, um die Steine zu fixieren und gleichzeitig zur Ausrichtung drehbar zu lagern. ${ }^{20}$ Von einem weiteren Nachvollzug dieses Verfahrens wurde abgesehen, um die Rohform des Gewölbes nicht durch Dübel zu verunklären, denn bildkünstlerische Ausgestaltungen und Applikationen waren vorerst nicht geplant.

Anschließend mussten, durch Drehung in der Horizontalen, alle Kreuzpunktsteine auch untereinander ausgerichtet werden. Auch hier halfen einfache Mittel und Verfahren des Spätmittelalters viel wei-

19 Vgl. Simon Garcia: Compendio de Architectura y Simetria de los Templos, 1681, fol. 25r (Seite 24); Foto: Ms. 8884, Biblioteca National de Espana, www.bne.es, Zugriff 16.04.2016.

20 Beispiele: St. Marien Pirna, Schlosskirche Chemnitz, Marktkirche Halle u.v.m. Auch der Stich von David Konrad zeigt an allen Rippenkreuzungen applizierte Sterne, die an solchen Eisendübeln befestigt worden sein dürften.

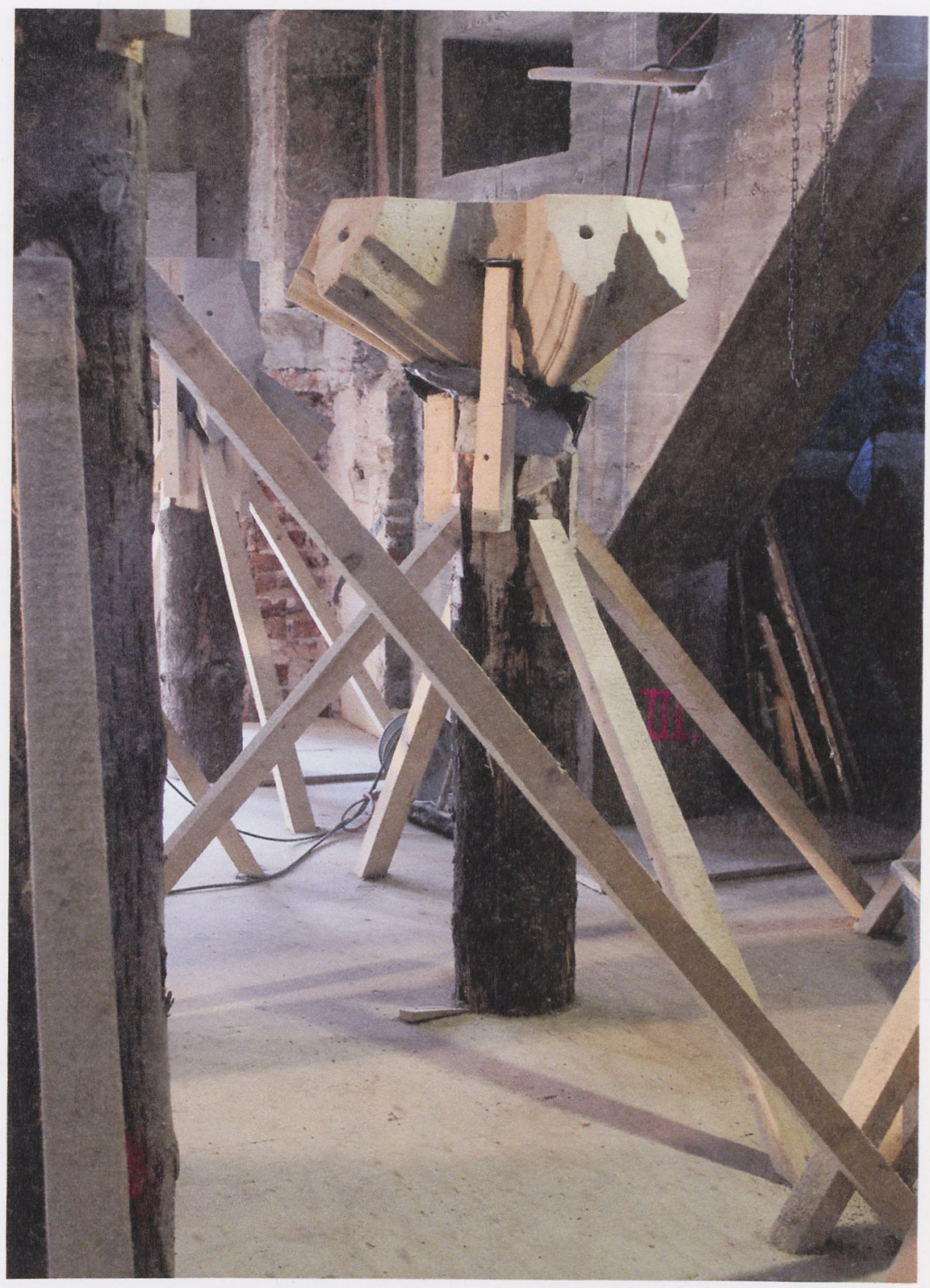

ter. Bei Schlosssteinen der Wappensaalwölbung auf der Albrechtsburg in Meißen war beobachtet worden, dass die Schlosssteine des Gewölbebinnenbereichs trotz ihrer leicht unterschiedlichen Höhe im Raum auf ihrer Rückseite ebene Oberflächen erhielten, die zum einen exakt waagerecht sind und zudem diese Flächen auf gleicher Höhe lagen. Auf den planen Oberseiten waren Linien aufgerissen, die exakt den Verbindungslinien zwischen zwei Kreuzpunkten entsprachen. Diese Flächen ließen sich mittels Setzwaage (Lotwaage, heute Wasserwaage) leicht so ausrichten, dass die Werkstücke nicht im Raum kippten. Mit den Risslinien konnten mittels Richtscheit und/oder Richtschnüren die Steine untereinander ausgerichtet werden,
Bild 17

Lehrgerüst für den Versatz der Rippenknoten aus unbesäumten Baumstämmen mit Zwickelstempel zur exakten Positionierung und Haltung nach Vorgabe von Höhen- und Lagepunkten der steintechnischen Werkplanung gemäß der Vorgaben von Bauer/Lauterbach [Foto: Thomas Bauer] 


\section{Bau des Rippenwerks, Bauprozess}

Nach erfolgreichem Belastungstest und Auswertung zum Probejoch wurde das Gewölbe jochweise errichtet (Bild 20): Der Arbeits- und Reißboden in Höhe der Wölbanfänger wurde dem Vorschlag von Thomas Bauer folgend als Stahlrahmenkonstruktion ausgebildet sowie ein Andienen der Werksteine von oben mit einen bautemporären, fahrbaren Stahl-Portalkran vorgesehen. Beide vorgenannten Hilfsmittel weichen gegenüber hölzernen Vorbildern des 15./16. Jahrhunderts zweifellos ab, die Prinzipien, mit innen ein Gewölbe zu bauen, sind aber die gleichen.

Eine Fertigungsfolge bestand aus folgenden Phasen: a) Übertragung des Wölbrisses auf dem Reißboden, b) Bau der Lehrkonstruktion über den Kreuzpunkten, c) Einlassen der Auflagersteine in die Pfeiler und Mauern,

d) Versetzen und Ausrichten der Schlosssteine, e) dann Versetzen und Vermörteln der Rippensteine.

Im Unterschied zu vielen Gewölben des 15. und 16. Jahrhunderts wurden die Steine untereinander nicht mit handgeschmiedeten oder hölzernen kantigen Dollen verdübelt ${ }^{21}$, sondern mit runden geriffelten Edelstahlbolzen. ${ }^{22}$ Dafür sind keine quadratischen Dübellöcher in die Fugenseiten der Werkstücke eingemeißelt worden, sondern Bohrungen angelegt. Auch wurden dann diese Dübel nicht mit Blei verlassen, sondern mit Mörtel vergossen. Zur zusätzlichen statischen Sicherung sind auf die Dübel gelochte Metallbänder aufgefädelt worden,

21 Es gibt genauso eine signifikante Anzahl bedeutender und großer spätgotischer Gewölbe, deren Befunde eine dübellose Verbindung der Steinrippen belegen, so u. a. St. Peter und Paul in Melnik/Böhmen von 1486 sowie der überwiegende Teil aller Gewölbe der Burg Ofen in Buda um 1500; siehe dazu: Bauer, Thomas; Lauterbach, Jörg; Nußbaum, Norbert: Die Königssäle Wladislaws II. in Buda und Prag. Erörterungen über Benedikt Rieds Beitrag zur Hofkunst der Jagiellonen. In: INSITU, Zeitschrift für Architekturgeschichte, 10. Jg. Heft 2, 2018, S. 227 - 242, hier S. 232 und Anm. 39.

22 Die Ausführung von Rippendübeln folgte dabei - unabhängig der Dübelform - der in Pirna an der spätgotischen Wölbung 1500 festgestellten Schleifdübeltechnologie (heutzutage auch als Ziestifte bezeichnet), vgl. Albrecht Sturm: Kapitel „Kunst der Fuge“. In: Sturm, Albrecht (Hrsg.): Die Stadtkirche St. Marien zu Pirna, Pirna 2005, S. $41-42$. die dann aus rein vorsorglichen Gründen in das spätere Kappenmauerwerk eingebunden wurden, um zukünftig ein mögliches Ablösen und Herabfallen von Rippenstücken ausschließen zu können. ${ }^{23}$

\section{Jochweises Bauen eines Gewölberippenwerkes}

Eine der großen Fragen zum Bau spätgotischer, figurierter Gewölbe stand seit Anfang der steintechnischen Werkplanung und Planung des Lehrgerüstes im Raum: Wie sind die Werkleute im Mittelalter mit dem Bauzustand zwischen verschiedenen Arbeitsständen mehrerer Joche - insbesondere dem Gewölbeschub zwischen zwei Jochen - bautemporär umgegangen? An den umfassenden Seitenwänden konnte der Gewölbeschub durch die zuvor gemauerten aufgehenden Außenwände mit Strebepfeilern (oder Wandpfeilerkirchen mit innenliegender Schubaufnahme) aufgenommen werden. Wo aber wurde während des Baues - als z. B. Joch 3 fertig war und mit Joch 4 erst begonnen wurde - der Gewölbeschub bautemporär zwischen den Jochen abgeleitet? Oder gar die Frage, kann man ein Joch fertig bauen ohne das benachbarte Joch begonnen zu haben? Mittelalterliche Quellen zu Beschreibungen von Bauabläufen bei Wölbungen sind rar und unpräzise, vermitteln aber gleichlautend die Vorstellung, dass im Verlauf von Wölbarbeiten auch Rüstarbeiten bspw. mit „bogstellen“ durchgeführt wurden. ${ }^{24}$ Aber in keiner bislang bekannten Quelle kann man sinngemäß etwas zu solchen temporären Bauzuständen oder statischen Verhältnissen wie Schubaufnahmen lesen. Die Problematik beim jochweisen Bauen eines Gewölbes besteht darin, dass wenn z. B. Joch 3 für sich allein gebaut wird (was bedeutet, dass zur Schubaufnah-

23 Das teilweise Lösen und Abfallen von einzelnen Rippen an spätgotischen Gewölben ist ein gängiges Schadbild; letztendlich bestätigt dies, dass das Kappenwerk tragender Teil der Wölbung ist und der Rippen im Endtragzustand nicht bedürfen. Beispielgebend dazu der Ratssaal Löwenberg/Lwowek Slaski vor seiner Sanierung in den 1960er Jahren. Fotos belegen die Wölbung mit bis auf die Anfänger vollständig abgefallenen Rippen; siehe Bauer/Lauterbach 2011 (wie Anm. 13), S. 53.

24 Dazu ist ein Projekt zu den überlieferten Beschreibungen zum sog. Kleinen Chor der Schlosskirche Wittenberg seitens der Autoren in Arbeit. 
Bild 20 Gesamtaufnahme des wiederhergestellten Schlingrippengewölbes der Schlosskapelle in

Dresden mit neuzeitlichem Arbeitsboden gemäß Sondervorschlag von Thomas Bauer;

Das Stahlrahmengerüst ist bereits teilweise zurückgebaut.

[Foto: Rainer Böhme]

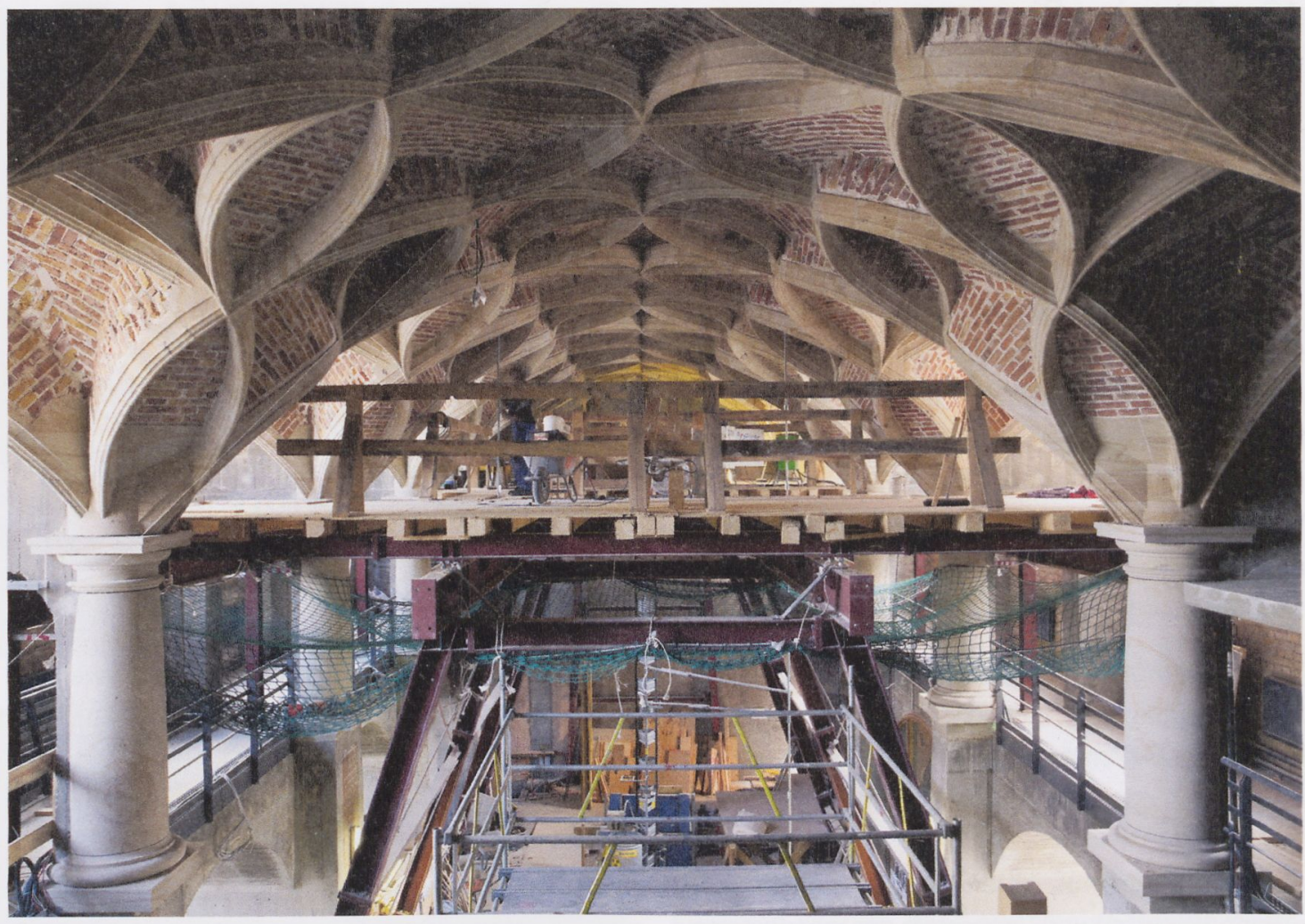

me Strebestützen ins Feld von Joch 4 gesetzt werden müssten) und danach das Lehrgerüst ins Joch 4 umgesetzt wird, müsste ein Teil des Gerüstes zur Schubaufnahme von J3 zu J4 (nämlich die schubaufnehmenden Strebestützen) stehen bleiben und würde Lehrgerüst und Arbeiten am J4 behindern. Zudem reifte im Verlauf die Erkenntnis, dass der Gewölbeschub sich im Wesentlichen erst dann aufbaut, wenn die Mauerwerkswölbung über die versetzten Rippen geführt wird, da für das Dresdner Gewölbe bspw. das Lastverhältnis Mauerwerksschale/Rippenwerk ca. 4: 1 beträgt. Aus diesen beiden Überlegungen heraus wurde die Technologie abgeleitet, dass zum Rippenversatz immer mindestens das nächste Joch mit Holzlehrgerüst bereits gebaut sein musste (zur Schubaufnahme aus dem Sandsteinrippenwerk) sowie zur Mauerwerkswölbung immer mindestens das nächste Joch mit Rippenwerk (und dem folgend auch das übernächste mit Holzlehrgerüst) versetzt und vergossen, d. h. verdübelt sein muss. Dass dies hypothetisch auch für die Wölbbauweisen des 15./16. Jahrhunderts anzunehmen ist, erschließt sich allein rückwirkend aus der Erfahrung, dass ein jochweises Einrüsten und Einwölben (Lehrgerüstbau und Rippenversatz) nicht funktionieren kann.
Auf die frei gewölbten - d.h. ohne flächige Holzschalungen gemauerte - Gewölbekappen aus Handstrichziegeln und auf der Baustelle angerührten Kalkspatzenmörtel wird in diesem Beitrag zur Natursteintagung 2019 nicht weiter eingegangen, da es den Rahmen dieses Beitrags sprengen würde. ${ }^{25}$

\section{Die Fertigung der Werksteinrippen}

Die Fertigung von Werksteinrippen und Rippenknoten als zweifach gekrümmte Schlingrippen stellte den spannendsten Teil der Rekonstruktionsarbeiten dar (Bild 16). Basis bildete die Dissertation von Stefan Bürger ${ }^{26}$ welche den bisherigen, vor allem auf den Vorarbeiten von Werner Müller basierenden Forschungsstand zusammenfasste sowie eigene Folgerungen aufzeigte. ${ }^{27}$ Diesbezüg-

25 Bauer, Thomas; Neuling, Kay; Huber, Ralf: Das Freie Wölben über den Schlingrippen der Schlosskapelle Dresden, In: Mauerwerk 18 (2014) Heft 5, S. $314-322$.

26 Bürger 2007 (wie Anm. 18).

27 Werner Müller; Norbert Quien, wie Anm. 14; Werner Müller 1974: Einflüsse der österreichischen und der 
lich war interessant, dass erste Versuche der praktischen Steinplanung - noch ohne Studium der Forschung - letztendlich zum ähnlichen Ergebnis kamen, wie zuvor von Werner Müller und Stefan Bürger beschrieben. Dies betraf insbesondere die formbestimmenden Ableitungen der Rippenrohkörper aus dem Grund- bzw. Wölbriss. Demnach wird der Rippenkörper im Grundriss von einem Mantelring eines Zylinders - in Breite des Rippenkörpers - umschrieben, so dass im ersten Schritt (gemäß historischer Fertigung) der Mantelring aus dem Rohblock geschlagen wurde. Danach erfolgte jeweils auf der gekrümmten Ansichtsseite des Mantelringes der Auftrag der Bogenaustragung für das betreffende Rippenteilstück.

Diesbezüglich gehen bis heute die Meinungen auseinander: Während Werner Müller davon ausging, dass einstmals eine gekrümmte biegbare Schablone zur Bogenaustragung auf den Mantelring aufgelegt wurde, um den Bogenverlauf anzureißen, ging die Steinplanung (Bauer/Lauterbach) davon aus, einzelne Höhenpunkte aus dem Riss der Bogenaustragung abzugreifen und auf den Mantelring ab- bzw. aufzutragen. Zudem wird diese Frage zum Auftrag der Bogenaustragung auf den Mantelring überlagert von der Frage, ob die Bogenaustragung als ,Abwicklung' oder als ,Orthogonalprojektion' konstruiert wurde. Werner Müller wies zwar schon 1974 nach, dass der Maßunterschied nur ca. $1,6 \%$ beträgt und so im Rahmen anderer feststellbarer Toleranzen (z. B. Fertigung/Versatz) lag. ${ }^{28}$ Der Diskurs betrifft im Wesentlichen die Frage, wie die einzig bekannte Textquelle zur Fertigung einer „gewundenen Reihung“ - Lorenz Lechlers Unterweisungen an seinen Sohn von 1516 - zu interpretieren und bautechnisch umzusetzen wä-

böhmisch-sächsischen Spätgotik in den Gewölbemustern des Jacob Facht von Andernach, In: Wiener Jahrbuch für Kunstgeschichte XXVII 1974, S. 65-82; Werner Müller; Norbert Quien: Spätgotik Virtuell, Weimar 1999; Werner Müller; Norbert Quien: Erdachte Formen Errechnete Bilder, Weimar 2000; Werner Müller: Steinmetzgeometrie zwischen Spätgotik und Barock, Petersberg 2002.

28 spätere Befunde - siehe hier insbesondere die laufenden Forschungen Norbert Nußbaum, Thomas Bauer, Jörg Lauterbach auf dem Burgberg in Budapest an ca. 300 ausgegrabenen spätgotischen Rippenwerkstücken - zeigen eben auch das es u.E. keine einheitliche sondern mehrere verschiedene Herstellverfahren gab, was anhand von Risslinien nachweisbar ist. re. ${ }^{29}$ Diese Frage wird die Forschung auch weiterhin beschäftigen. ${ }^{30}$

Das Ziel, sowohl historische Rippenwerksformen nachzubilden und dabei bestenfalls auch Kosten zu senken, war nicht nur eine für die Dresdner Rekonstruktion spezifische Vorgabe, ${ }^{31}$ sondern steht regelmäßig bei einer Reihe angedachten Rekonstruktionen historischer Steinkonstruktionen an.

Diesbezüglich brachte dieser Wiederherstellungsprozess des Dresdner Schlosskapellengewölbes einen doppelten Gewinn: Zum einen konnten durch das praktische Verfahren zusätzliche Erkenntnisse gewonnen werden, die in nachfolgende wissenschaftliche Forschungen einflossen, zum anderen erwies sich, dass sich durch die Neuorientierung zeitgemäßer Technologien auf historische solche derartige Tragwerkkonstruktionen durchaus kostensenkend ,rekonstruieren', d.h. dem Verfahren nach neu herstellen ließen.

29 Coenen, Ulrich: Die spätgotischen Werkmeisterbücher in Deutschland, München 1990, S. 15 - 24 und 174- 266.

30 Zum Vergleich der zwei verschiedenen Fertigungsansätze von Schlingrippen inkl. bebilderter Darstellung zu den Fertigungsabfolgen: siehe Bauer; Lauterbach 2011 (wie Anm. 13), kostenfreier Download: www.schlingrippe.de/Publikationen.

31 Aktuelle Veröffentlichungen beschreiben die Herangehensweise der Dresdner Steinplanung unzutreffend: „.... wurden die Rippen mit einem parametrischen Modell abgebildet ..." sowie „... Dieses moderne, am Computer realisierte Beschreibungsmodell wurde verwendet, weil das spätgotische Verfahren der Steinplanung damals noch nicht beschrieben war ...", vgl. Wendland, David: Architekturgeschichte live. In: Bürger, Stefan; Kallweit, Ludwig (Hrsg.): Capriccio \& Architektur - Das Spiel mit der Baukunst, Berlin/München 2017, S. 117-125. Die computergestütze Steinplanung am 3D-Modell für das Dresdner Schlosskapellengewölbe erfolgte einzig mit dem Ziel, die Rohblockgrößen der Steinrippen signifikant zu minimieren und die Kosten im avisierten Rahmen zu senken. Traditionell gefertigte Rippen werden von großen Rohblöcken ausgehend aus vertikalen und horizontalen Flächen umschrieben; hingegen das Dresdner Verfahren konnte geneigte Rippen (entsprechend Einbaulage) auch in kleineren Rohblöcken umschreiben und mittels sechsseitigen Schablonen fertigen. 